\title{
The value of health insurance: the access motive
}

\author{
John A. Nyman * \\ Division of Health Services Research and Policy, University of Minnesota, 420 Delaware St. SE, Box \\ 729, Minneapolis, MN 55126, USA
}

Received 31 August 1997; revised 1 June 1998; accepted 24 June 1998

\begin{abstract}
Why do people purchase health insurance? Many economists would answer that it permits purchasers to avoid risk of financial loss. This note suggests that health insurance is also demanded because it represents a mechanism for gaining access to health care that would otherwise be unaffordable. For example, although a US $\$ 300,000$ procedure is unaffordable to a person with US $\$ 50,000$ in net worth, access is possible through insurance because the annual premium is only a fraction of the procedure's cost. The value of insurance for coverage of unaffordable care is derived from the value of the medical care that insurance makes accessible. (C) 1999 Elsevier Science B.V. All rights reserved.
\end{abstract}

JEL classification: I10; D81; D61

Keywords: Access motive; Health insurance; Expected utility

Why do people purchase health insurance? One standard response to this question is: to avoid financial risk. This response is based on expected utility theory and the premise that people generally prefer certainty to risk (Von Neumann and Morgenstern, 1944; Friedman and Savage, 1948). According to this theory, if consumers are sufficiently averse to financial risk, firms selling insurance can charge premiums that cover the expected coverage losses plus a loading fee, and still sell insurance.

\footnotetext{
*Tel. +1-612-626-4425; Fax: +1-612-624-2196; E-mail: nyman001@tc.umn.edu
} 
In a response to the argument of Arrow (1963) that health insurance is generally desirable because of this risk-avoidance feature, Pauly (1968) noted that moral hazard could create such a large net utility loss that insurance would become undesirable. Thus, in order for insurance to be purchased, the risk-avoidance benefit must exceed both the loading fee and the moral hazard loss.

Several authors have attempted to estimate the relative magnitudes of the risk-avoidance gain and the moral hazard loss of health insurance coverage in the U.S. (Feldstein, 1971, 1973; Friedman, 1974; Feldman and Dowd, 1991; Manning and Marquis, 1989, 1996). Using some variant of the measure of Pratt (1964) for the risk-avoidance gain, these studies found that moral hazard losses outweigh the gains from financial risk-bearing. For example, using data from the Rand Health Insurance Experiment, Feldman and Dowd (1991) conclude that when a free fee-for-service policy is substituted for a similar policy with a US\$1000 deductible, $^{1}$ the moral hazard loss is 10 times the risk-avoidance gain (US\$1795 v. US\$176, respectively, per family in 1984 dollars). Also using Rand data, Manning and Marquis $(1989,1996)$ show that if a $1 \%$ coinsurance rate policy is substituted for a $100 \%$ coinsurance rate policy (self-insurance), the moral hazard loss is over two times the risk-avoidance gain (US\$2553 v. US\$1130, respectively, per family in 1995 dollars). If these findings reflect the true relative costs and benefits of insurance, then it would be difficult to see why unsubsidized health insurance is purchased at all. ${ }^{2}$

This paper makes the case that the benefits from health insurance are not limited to the gain from avoidance of financial risk by the risk averse purchaser. Additional benefits are derived from insurance's ability to make available medical care that would not otherwise be affordable. This access value of insurance is different from the risk-avoidance value because, by definition, there is no financial risk for unaffordable health care purchases because the purchases cannot privately occur. That is, insurance is often the only mechanism available for gaining access to expensive health care: there may not be enough time (or income) to save for an expensive health care purchase, and lending institutions may be reluctant to lend money for health care procedures when the ability of the patient to repay these uncollateralized loans is limited. If insurance is the only way to gain access to expensive health care, then the value of insurance for that care is the expected

\footnotetext{
${ }^{1}$ This refers to the $95 \%$ coinsurance policy with the US $\$ 1000$ stoploss provision.

${ }^{2}$ Some analysts attribute the fact the people still purchase health insurance to the tax subsidy that allows employees to purchase insurance with pre-income tax dollars, but the effect of the tax subsidy is not large for the average worker. The federal median marginal income tax rate of $22.65 \%$ barely offsets the average loading fee, estimated at $18 \%$ in 1990 for commercial firms selling group insurance (Phelps, 1997). Thus, explaining the purchase of insurance by persons living in states without an additional state income tax is still problematic.
} 
consumer surplus from the health care services that would otherwise be inaccessible. This value may be large, especially if the procedure that insurance made possible were life-saving.

In what follows, the distinction between the access motive and the risk-avoidance motive is made with the aid of a simple model. Then, an estimate of the proportion of a typical insurance premium devoted to the access motive is presented. Measures of the access value of health insurance are then proposed, and a lower bound estimate of the access value of health insurance is suggested. In Section 5, charity and alternative sources of access to unaffordable care are considered.

\section{The access motive}

Consider a consumer who derives utility from disposable wealth $(w)$ and from health $(H) . H$ is a function of medical care $(M)$, and the utility of $w$ and $M$ differs according to whether you are sick, s, or healthy, h, so that:

$$
\begin{aligned}
& u^{\mathrm{s}}=u^{\mathrm{s}}(w, M) \text { and } \\
& u^{\mathrm{h}}=u^{\mathrm{h}}(w, M),
\end{aligned} .
$$

where $u_{w}>0$ and $u_{w w}<0$. Assume that $u_{M}^{\mathrm{s}}>0$ but $u_{M}^{\mathrm{h}}=0$ because, at best, medical care received when healthy does no good. Assume further that the only way to obtain $M$ is to purchase it privately out of the consumer's wealth or through the payoff from insurance.

With insurance, $w=W-M-P+I$, where $W$ is gross wealth, $P$ is the insurance premium, and $I$ is the insurance payoff. The probability that the sick state of the world would occur is $\pi$, where $0<\pi<1$. Insurance is assumed to be fair, so $\pi M=P$, and only comprehensive policies are available, so $I=M$. Assume that the price of medical care is fixed so that $M$ is normalized.

Assume that insurance can be purchased for different diseases, cafeteria style. Diseases have standardized treatment protocols. Some treatment protocols have costs, $M$, that are less than gross wealth, $W$, and some have costs that exceed gross wealth. When treatment costs exceed gross wealth, the treatments cannot be purchased, but insurance coverage for such medical care can be purchased because $P(=\pi M)<W$.

Consider first insurance for a disease for which $M<=W$. The gain from insurance for a specific illness is approximated by the traditional risk-avoidance gain that has been identified and incorporated into previous calculations:

$$
\mathrm{EU}=\pi u^{\mathrm{s}}[W-M, M]+(1-\pi) u^{\mathrm{h}}[W, 0]
$$


without insurance, and

$$
\begin{aligned}
\mathrm{EU} & =\pi u^{\mathrm{s}}[W-M-P+I, M]+(1-\pi) u^{\mathrm{h}}[W-P, 0] \\
& =\pi u^{\mathrm{s}}[W-P, M]+(1-\pi) u^{\mathrm{h}}[W-P, 0]
\end{aligned}
$$

with insurance. Without insurance, the consumer risks losing $M$ with the probability of $\pi$, but with insurance, the consumer is assured of losing only $P$. The only difference between this model and the conventional model is that utility differs according to health state. If $u^{\mathrm{s}}=u^{\mathrm{h}}=u$, the value of insurance for such treatment protocols would be the traditional risk premium measure.

Now, consider an illness where the standard treatment protocol's cost, $M$, exceeds endowed wealth, $W$. For example, a consumer might have US\$50,000 in net worth from all sources and be considering the purchase of insurance coverage for a liver transplant that costs US\$300,000 for the entire treatment protocol. If diagnosed, there might be no time or ability to save the extra amount out of income, and lending institutions are unlikely to write a US\$250,000 (the difference between the cost of the procedure and what the patient is able to raise by selling all his assets) uncollateralized loan because of the riskiness of the procedure and possibility that the loan will not be repaid. For health care purchases like this, there might be no private mechanism for gaining access to this procedure if ill, save for already having purchased health insurance coverage.

The consumer derives utility from the same two arguments, but without insurance, expected utility is

$$
\mathrm{EU}=\pi u^{\mathrm{s}}[W, 0]+(1-\pi) u^{\mathrm{h}}[W, 0] .
$$

because no loss of $M$ dollars from disposable wealth occurs if the consumer does not have at least $M$ dollars to spend. More importantly, in comparison to Eq. (1), Eq. (3) shows there is no expected utility gain from the medical care because this procedure is not available without health insurance. With insurance, expected utility is

$$
\begin{aligned}
\mathrm{EU} & =\pi u^{\mathrm{s}}[W-M-P+I, M]+(1-\pi) u^{\mathrm{h}}[W-P, 0] \\
& =\pi u^{\mathrm{s}}[W-P, M]+(1-\pi) u^{\mathrm{h}}[W-P, 0]
\end{aligned}
$$

Comparing Eqs. (3) and (4) reveals that the gain in utility from insurance is access to $M$ expenditures of medical care in the event of illness, at a cost of $P$ dollars of disposable wealth. Thus, the net gain from insurance coverage of otherwise unaccessible medical care is approximated by the expected consumer surplus from that medical care.

Fair insurance enables the consumer to gain access to procedures whose costs exceed the consumer's wealth because the consumer need only pay the actuarially fair premium, which is the product of the cost of the treatment and the probability of becoming ill. Thus, insurance allows consumers to gain access to many procedures that they would not otherwise be able to purchase. For example, the 
probability of requiring a US\$300,000 liver transplant was about $1.33 / 100,000$ a year in 1992. ${ }^{3}$ Without insurance, access to such a procedure would have required US $\$ 300,000$ in resources. With insurance, it could have been obtained by paying (US\$300,000*1.33/100,000 =) US\$4 annually for a fair insurance policy. The access value of that policy could be estimated by subtracting the US\$4 premium from the expected value of that liver transplant.

\section{The prevalence of the access motive}

How prevalent is the access motive? A rough estimate of the prevalence of the access motive can be derived from the distribution of wealth among the population of the U.S. and the distribution of health care expenditures.

The Federal Reserve Board's Survey of Consumer Finances (Kennickell and Shack-Marquez, 1992) estimates that the median net worth in 1989 was US\$47,200. The National Medical Expenditure Survey (NMES) in 1987 estimates that the top spending $1 \%$ of the population accounted for $30 \%$ of the total spending. The average annual individual expenditure for this $30 \%$ of total spending was US\$47,331 (Berk and Monheit, 1992). Comparing this figure with the median net worth suggests that, for the person at the median of the wealth distribution, about $30 \%$ of that person's health insurance premium is devoted to expenditures that would exceed the net worth of his or her household. ${ }^{4}$ For persons from wealthier households, that percentage would be lower, but for persons from poorer households, the percentage would be higher.

The prevalence of the access motive indicates the extent to which existing estimates of the value of insurance are too low. That is, the value of access to unaffordable medical procedures should be estimated from the expected consumer surplus from these procedures, and included in the estimates of the value of health

\footnotetext{
${ }^{3}$ The mean total inpatient charges for a liver transplant in 1992 was estimated at US\$304,567 according to the Healthcare Cost and Utilization Project's Nationwide Inpatient Sample (U.S. Agency for Health Care Policy and Research, 1998). Also according to that study, there was an estimated 2964 liver transplants done on non-Medicare patients in the U.S. in 1992. The U.S. population younger than 65 was estimated at $222,729,000$ (U.S. Bureau of the Census, 1997). A rough estimate of the annual liver transplant rate in 1992 is therefore about 1.33/100,000.

${ }^{4}$ It should be noted that the $30 \%$ figure is only a first approximation. Many adjustments would need to be made to obtain a refined estimate. For example, the elderly on Medicare would need to be excluded in order to obtain an estimate of this percentage for private insurance. Although the elderly make up a disproportionate share of the top spenders, they also have higher net worths. Therefore, it is unclear what the net effect of excluding the elderly would be. Another refinement would need to account for those who are able to obtain some level of treatment with existing resources, but not, perhaps, the preferred treatment. Still, another adjustment would need to be made for those persons with seemingly affordable expenditures who have chronic diseases: such persons would perhaps be able to cover this year's expenditures from net worth, but would find the required treatments unaffordable over a longer time period.
} 
insurance. The prevalence of the access motive may also indicate the extent to which some existing estimates of the risk-bearing value of insurance are too high. That is, if an expenditure is not affordable, there is no risk of losing it. If such expenditures are used to calculate the financial risk-avoidance value of insurance, they should be omitted and the estimates of the value of risk-avoidance should be adjusted downward.

\section{Estimating the access value of insurance for a given procedure}

The access value of insurance is related to the value to the consumer of medical care that the person would not otherwise be able to afford. There are a number of potential approaches for estimating the value to the consumer of expensive medical procedures.

One way would be to estimate demand for a high cost procedure among wealthy consumers who are uninsured and calculate the area under the demand curve. Because few wealthy individuals are uninsured (Lefkowitz and Monheit, 1991), obtaining data sufficient to estimate the demand for the treatment for high cost medical procedures would be problematic.

When demand data are not available, an alternative approach is to identify a sample of persons and ask them hypothetical questions of what their willingness to pay would be for each type of procedure. Because many of these procedures are beyond the resources of many of the respondents, it would be necessary to ask the question in an insurance frame: What would the respondent be willing to pay for an annual insurance policy covering treatment of disease $X$, given that there is only a $Y / 100,000$ chance that the respondent would need that treatment in the coming year? It is well-known that one problem with hypothetical questions is the possibility of strategic answering. It would, therefore, be preferred to rely on actual market behavior to determine these values.

A third approach to estimating the value of otherwise unaffordable medical care is to use the value of the outcome of the medical care to the patients. One outcome that has been extensively explored in the literature is the value of a human life. That is, for medical care that saves lives, the value of insurance that makes such medical care possible is the value of a life saved. Thus, the willingness to pay for a life-saving medical procedure can, therefore, be approximated by the value of a statistical life. Viscusi (1993) reviews the value of life literature-much of which is based on actual market decisions - and concludes that an estimate in the US\$3 million to US\$7 million range (in 1990 dollars) is most defensible.

Less explored is the value of an added year of life or an added quality adjusted life year (QALY). The use of the value of a QALY has the advantage that it could measure the value of care that either extended the number of years of life or improved the quality of a given life expectancy because the care cured or improved quality of life of a person suffering from a non-fatal disease. Some measures attempt to estimate this value from the results of the value of life 
literature (Tolley et al., 1994; Johannesson and Meltzer, 1998). A reasonable estimate of the value of a year of life or a QALY may be US\$100,000.

For example, for an individual who is contemplating the purchase of insurance coverage for a liver transplant operation but who does not have the private resources to cover its costs, the access value of insurance for a liver transplant can be calculated as follows. As noted there is about a 1.33/100,000 chance that a person would have a liver transplant performed in a given year. For transplants done over 1982-1991, 69.2\% of recipients lived at least 5 years (the 5-year survival rate) after their liver transplant (Kilpe et al., 1993), and the survival rates have been increasing over time (Busuttil et al., 1994). Also increasing over time has been the proportion of liver transplant recipients that are classified in the United Network for Organ Sharing (UNOS) criteria as 'status 4': patients who have less than 1 week to live or are already on life support when a transplant is performed. This type of patient represented only $11 \%$ of a sample of patients in 1982, but $37 \%$ in 1991 (Busuttil et al., 1994). The large and growing percent of transplant patients in this category supports the assumption that the life expectancy (or the quality of life for those patients on life support) of transplant patients without a transplant would be negligible, and that the years of life lived after a transplant represent a net gain.

About $30 \%$ of liver transplant recipients live less than 5 years and $70 \%$ live more than 5 years with no information on the upper bound. If it is assumed that most of the mortality risk from the transplant occurs from possible organ rejection in the initial years, it would be reasonable to assume that expected number of survival years after a transplant is at least 7. At a value of US\$100,000 per year of life saved, the consumer's evaluation of a liver transplant would be approximately $[(1.33 / 100,000)(7 * \$ 100,000)=] \mathrm{US} \$ 9.31 .{ }^{5}$ As already noted, the actuarially fair premium for a US\$300,000 liver transplant would be $[(1.33 / 100,000)$ (US\$300,000) = JUS\$4. If these values are accurate, the net value of a fair insurance policy that covers liver transplantation for a person who otherwise could not afford one is (US\$9.31 - US\$4 =) US\$5.31. For a health insurance policy that covers a number of otherwise unaffordable procedures, the access value would be the sum of these expected net values.

\section{Estimating the access value of health insurance in the US}

It is difficult to estimate the access value of health insurance in the US in the absence of a full-fledged empirical study. This paper is intended to identify the

\footnotetext{
${ }^{5}$ This crude accounting for the value of unaffordable care ignores discounting, variations in the marginal utility of income and how they may affect the evaluation of a QALY, and other issues that would be part of a full-fledged cost-benefit analysis.
} 
concept and suggest that its value is not inconsequential. One approach to establishing the potential importance of the access value of insurance in the absence of better information is to use NMES data to estimate a lower bound access value.

NMES data indicated that the top spending 1\% of the population in 1987 accounted for about $30 \%$ of all expenditures. The U.S. population in 1987 was about 240 million, so $1 \%$ was about 2.4 million. Half of the top spenders qualify under Medicaid or Medicare, so those with private insurance were about 1.2 million (Berk and Monheit, 1992).

As mentioned above, the Survey of Consumer Finances (Kennickell and Shack-Marquez, 1992) estimates that the median net worth in 1989 was US\$47,200. The NMES estimates that the top spending $1 \%$ of the population accounted spent on average US\$47,331 (Berk and Monheit, 1992). If health expenditures are distributed uniformly across the wealth distribution, this suggests that the half of the population with net worths less than US\$47,200 would not be able to afford these expenditures. Therefore, for about 600,000 of the 1,200,000, their expenditures would have been unaffordable save for insurance.

The estimate that 600,000 had unaffordable procedures in 1987 is conservative. Among those 600,000 with net worths above US\$47,200, some would not have been able to afford some of the procedures (for example, a person from a household with a US\$100,000 net worth would also not be able to afford a US $\$ 300,000$ procedure). These cases are not counted because it is not clear how many they represent. Similarly, among those 237.6 million with health care expenditures less than US\$47,331, about half or about 118.8 million would have had net worths less than US\$47,200. Some of these people would have had health care expenditures that exceeded their net worth, too (for example, a US\$40,000 procedure may have been unaffordable to a person with US $\$ 30,000$ in net worth). These cases are also not included. Therefore, to focus on the 600,000 cases that are supported by the NMES data understates the number affected.

Nevertheless, if each of the 600,000 persons receiving expensive medical care averaged, say, from 1 to 5 additional years of life (or 1 to 5 additional QALYs for those whose morbidity was lessened by the medical care), evaluated at US $\$ 100,000$ a year, this implies a value of US\$60 billion to US\$300 billion. After subtracting the cost of the medical care $(600,000$ persons at an average cost of about US\$50,000 each equals US\$30 billion), this implies a net gain of US\$30 billion to US $\$ 270$ billion. Thus, a reasonable estimate may be to assume the midpoint of this range or 3 years, and calculate that the lower bound access value of health insurance was US\$150 billion in 1987.

In comparison, Manning and Marquis (1989) estimate that for every family with a $1 \%$ coinsurance rate insurance policy, there is a US\$706 (1988 dollars) gain in value of risk-bearing from insurance compared with no insurance. For the $71,610,000$ U.S. households headed by a person under age 65 in 1988 (U.S. Bureau of the Census, 1998), the total value from risk-bearing in the U.S. would 
be about US $\$ 50.5$ billion. Thus, the lower-bound estimate of the access value (US\$150 billion) is about three times as large as the risk-bearing value.

The risk-bearing estimate based on Manning and Marquis' study is liberal because insured families typically confront a greater amount of cost-sharing in their insurance than the $1 \%$ coinsurance rate that these authors assume. Insurance does not cancel the risk of out-of-pocket expenditures. It is also liberal because the variance in expenditures that was used to estimate the value of risk-bearing was calculated from a range of expenditures that may include those that are unaffordable. Excluding the unaffordable expenditures from the data used to calculate the variance in expenditures would reduce the variance. This implies that the variance used to estimate the risk-bearing value is likely greater than the appropriate one, and that Manning and Marquis' estimate of the risk-bearing value of insurance maybe too large.

\section{Charity}

In the original model, it was assumed that the only ways to purchase health care were through one's own wealth or through insurance. Now relax this assumption by considering charity.

Charity represents one way that a person could gain access to health care without either purchasing it or through the access mechanism of insurance. Although charity is common in our economy, especially for those commodities like food, clothing and shelter which are considered necessities (in the non-technical sense of the word), it is rarely considered in analyses of the efficiency of these markets. In health care, however, charity has historically played such a major role that it cannot be ignored, especially in describing the counterfactual for this analysis, namely, the state of the economy without health insurance.

If the alternative of charity care is considered, the value of insurance again has both a risk-avoidance and an access value, but the access value is net of the value of services that could be obtained through charity and the likelihood of receiving them. Studies have shown that important differences exist between the characteristics and outcomes of care for the insured and uninsured (e.g., Franks et al., 1993; Young and Cohen, 1991), and that care is sometimes not available for the uninsured (e.g., Himmelstein and Woolhandler, 1995; Donelan et al., 1996). It would therefore be reasonable to conclude that these differences would still result in a positive access value of private health insurance.

It is also widely believed that for some consumers, charity has a social stigma associated with it. This stigma may further reduce the value of charity-financed care. Indeed, in some cases, the aversion to charity may be so intense that charity-financed care is not recognized as a viable alternative to insurance-financed care. Both these responses to charity would increase the access value of insurance. 
It is not clear, however, whether charity should be considered an alternative to private health insurance when estimating the value of all private insurance in the U.S. While it would be reasonable to view charity as an alternative for the exceptional individual, it would be unreasonable to view charity as an alternative for all the people who would not be able to afford the care they 'need' were it not for insurance. This care must be paid for in some way, and it is likely that charitable contributions would be exhausted quickly if they were required to cover all the unaffordable care that is insured. This implies that, from a societal perspective, there is a fallacy of composition argument that favors ignoring charity in estimating the access value of insurance. Similar arguments could be made for other mechanisms for gaining access to unaffordable care, such as through bad debts, public delivery systems, and even public insurance such as Medicaid.

\section{Conclusions}

The access motive is not new. Sheer affordability of health care was recognized as the prime reason for needing/desiring health insurance as early as 1927 with the creation of the national Committee on the Costs of Medical Care (Falk et al., 1933; Falk, 1936; Rorem, 1982). It was widely written about in the 1950s and 1960s (Anderson, 1956; Somers and Somers, 1961) and in the 1970s by some public figures (Kennedy, 1972). In the U.S. health care reform debate of 1994, the central issue was personified by individuals who were unable to obtain needed health care because they were uninsured. That economists have overlooked access in determining the value of health insurance is a puzzle.

This paper argues that in overlooking the access motive, economists have overlooked an important source of value in health insurance: the ability to provide access to procedures that would otherwise be unaccessible. The magnitude of the access value may far exceed the value of insurance for financial risk-bearing. Because people do purchase health insurance, the incorporation of the access value into the theory of health insurance may result in a theory that better conforms to actual behavior.

The value of insurance has traditionally been conceptualized with respect to the counterfactual of having the resources available to incur the loss in question, that is, of self-insurance. This is a reasonable counterfactual for determining the value of insurance for property losses, because the property was presumably acquired through the use of the person's resources in the first place and has a resale value after its purchase. With health insurance, the value that people place on their lives and health means that when ill, they may desire to incur expenditures for health care that exceed their resources. Because insurance is often a unique means for paying for these expenditures, the value of such health insurance is related to the value of health care that would otherwise be unobtainable. This paper has shown that this access value of health insurance is likely to be substantial. An explicit 
accounting for it would provide a more complete understanding of why people buy health insurance.

\section{Acknowledgements}

In writing this paper, I have benefitted from the comments of David Cutler, Bryan Dowd, Roger Feldman, Will Manning, Joe Newhouse, Carl Phillips, and an anonymous referee. The views in this paper, and any remaining errors or oversights, are my own.

\section{References}

Anderson, O.W., 1956. Family medical costs and voluntary health insurance: a nationwide survey. McGraw-Hill, New York.

Arrow, K.J., 1963. Uncertainty and the welfare economics of medical care. Am. Econ. Rev. 53, 941-973.

Berk, M.L., Monheit, A.C., 1992. The concentration of health expenditures: an update. Health Affairs 11, 145-149.

Busuttil, R.W., Shaked, A., Millis, J.M., Jurim, O., Colquhoun, S.D., Shackleton, C.R., Nuesse, B.J., Csete, M., Goldstein, L.I., McDiarmid, S.V., 1994. One thousand liver transplants: lessons learned. Annals Surgery 219, 490-499.

Donelan, K., Blendon, R.J., Hill, C.A., Hoffman, C., Rowland, D., Frankel, M., Ahanan, O., 1996. Whatever happened to the health insurance crisis in the United States? Voices from a national survey. J. Am. Medical Assn. 276, 1346-1350.

Falk, I.S., 1936. Security against illness: a study of health insurance. Doubleday, Garden City, NY.

Falk, I.S., Klem, M.C., Sinai, N., 1933. The incidence of illness and the receipt and costs of medical care among representative families: experiences in twelve consecutive months. Committee on the Costs of Medical Care report 26. Univ. of Chicago Press, Chicago.

Feldman, R., Dowd, B., 1991. A new estimate of the welfare loss of excess health insurance. Am. Econ. Rev. 81, 297-301.

Feldstein, M.S., 1971. Hospital cost inflation: a study in nonprofit price dynamics. Am. Econ. Rev. 61, 853-872.

Feldstein, M.S., 1973. The welfare loss of excess health insurance. J. Political Economy 61, 251-280.

Franks, P., Clancy, C.M., Gold, M.R., 1993. Health insurance and mortality: Evidence from a national cohort. J. Am. Medical Assn. 270, 737-741.

Friedman, B., 1974. Risk aversion and the consumer choice of health insurance option. Rev. Econ. Statistics 50, 209-214.

Friedman, M., Savage, L.J., 1948. The utility analysis of choices involving risk. J. Political Economy $56,279-304$.

Himmelstein, D.U., Woolhandler, S., 1995. Care denied: US residents who are unable to obtain needed medical services. Am. J. Public Health 85, 341-344.

Johannesson, M., Meltzer, D., 1998. Some reflections on cost-effectiveness analysis. Health Economics 7, 1-7.

Kennedy, E.M., 1972. In critical condition: the crisis in Americas's health care. Simon and Schuster, New York. 
Kennickell, A.B., Shack-Marquez, J., 1992. Changes in family finances from 1983 to 1989: evidence from the survey of consumer finances. Fed. Reserve Bull. 78, 1-18.

Kilpe, V.E., Krakauer, H., Wren, R.E., 1993. An analysis of liver transplant experience from 37 transplant centers as reported to medicare. Transplantation 56, 554-561.

Lefkowitz, D., Monheit, A., 1991. Health insurance, use of health services and health care expenditures (AHCPR Pub. No. 92-0017). National medical expenditure survey research findings 12. Agency for Health Care Policy and Research, Rockville, MD.

Manning, W.G., Marquis, M.S., 1989. Health insurance: the trade-off between risk pooling and moral hazard. Rand, Santa Monica, CA.

Manning, W.G., Marquis, M.S., 1996. Health insurance: the tradeoff between risk pooling and moral hazard. J. Health Econ. 15 (5), 609-640.

Pauly, M.V., 1968. The economics of moral hazard: comment. Am. Econ. Rev. 58, 531-537.

Phelps, C.E., 1997. Health economics, 2nd edn., Harper Collins, New York.

Pratt, J.W., 1964. Risk aversion in the small and in the large. Econometrica 32, 122-136.

Rorem, C.R., 1982. A quest for certainty: essays on health care economics 1930-1970. Health Administration Press, Ann Arbor.

Somers, H.H., Somers, A.R., 1961. Doctors, patients and health insurance. Brookings, Washington, D.C.

Tolley, G., Kenkel, D., Fabian, R., 1994. State-of-the-art health values, In: Tolley, G., Kenkel, D., Fabian R. (Eds.), Valuing Health Policy: An Economic Approach. Univ. of Chicago Press, Chicago, pp. 323-344.

U.S. Agency for Health Care Policy and Research, Department of Health and Human Services. National statistics by diagnosis-related group: HCUP-3 nationwide inpatient sample for 1992 hospital inpatient stays. Agency for Health Care Policy and Research website, 1998.

U.S. Bureau of the Census, Current population reports, Series household and family characteristics: March 1996 (update). Households, by age of householder: 1960 to present. Bureau of the Census website, 1997.

U.S. Bureau of the Census, Population Division. Release PPL-91. United States population estimates, by age, sex, race, and Hispanic origin, 1990 to 1997. Bureau of the Census website, 1998.

Viscusi, W.K., 1993. The value of risks to life and health. J. Econ. Literature 31, 1912-1946.

Von Neumann, J., Morgenstern, O., 1944. Theory of games and economic behavior. Princeton University Press, Princeton, NJ.

Young, G.J., Cohen, B.B., 1991. Inequities in hospital care, The Massachusetts experience. Inquiry 28, $255-262$. 